\title{
Lysophosphatidic Acid Promotes Epithelial to Mesenchymal Transition in Ovarian Cancer Cells by Repressing SIRT1
}

\author{
Upasana Ray ${ }^{a}$ Shreya Roy Chowdhury ${ }^{a}$ Sib Sankar Roy ${ }^{a, b}$ \\ ${ }^{a}$ Cell Biology and Physiology Division, CSIR-Indian Institute of Chemical Biology, Kolkata, ${ }^{b}$ Academy of \\ Scientific and Innovative Research, CSIR- Indian Institute of Chemical Biology Campus, Kolkata, India
}

\author{
Key Words \\ EMT • Lysophosphatidic acid $\bullet$ SIRT1 • Ovarian cancer
}

\begin{abstract}
Background/Aims: Epithelial-to-mesenchymal transition (EMT) plays an essential role in the transition from early to invasive phenotype, however the underlying mechanisms still remain elusive. Herein, we propose a mechanism through which the class-III deacetylase SIRT1 regulates EMT in ovarian cancer (OC) cells. Methods: Expression analysis was performed using Q-PCR, western blot, immunofluorescence and fluorescence-IHC study. Matrigel invasion assay was used for the invasion study. Morphological alterations were observed by phalloidin-staining. Co-immunoprecipitation study was performed to analyze protein-protein interaction. Results: Overexpression of SIRT1-WT as well as Resveratrol-mediated SIRT1 activation antagonized the invasion of OC cells by suppressing EMT. SIRT1 deacetylates HIF1 $\alpha$, to inactivate its transcriptional activity. To further validate HIF1 $\alpha$ inactivation, its target gene, i.e. ZEB1, an EMTinducing factor was found to attenuate upon SIRT1 activation. To uncover the regulatory factor governing SIRT1 expression, lysophosphatidic acid (LPA), a highly enriched oncolipid in ascites/ serum of OC patients, was found to down-regulate SIRT1 expression. Importantly, LPA was found to induce the mesenchymal switch in OC cells through suppression of SIRT1. Decreased level of SIRT1 was further validated in ovarian tissue samples of OC patients. Conclusion: We have identified a mechanism that relates SIRT1 down-regulation to LPA-induced EMT in OC cells and may open new arenas on developing novel anti-cancer therapeutics.
\end{abstract}

\section{Introduction}

(C) 2017 The Author(s)

Published by S. Karger AG, Basel

Ovarian cancer (OC) emerges as a highly metastatic disease among all the gynaecological malignancies, mainly due to its asymptomatic nature followed by late detection and the aggressive phenotype [1]. Therefore, the present situation needs detailed understanding of the disease that will open up new therapeutic horizons to decrease the associated mortality rate. 
Metastatic potential of the cancer cells depends on the attainment of epithelial-tomesenchymal transition, which is a phenomenon characterized by breakdown of cell junctions and loss of cell polarity, rendering epithelial cells motile and invasive [2]. EMT plays a significant role in development, particularly in early embryogenesis; however, when aberrantly activated, results in cancer metastasis [3]. It is characterized by down-regulation of the genes commonly found in epithelial cells (e.g. E-cadherin, occludin) and the possession of genes typical to mesenchymal cells (e.g. N-cadherin, vimentin, fibronectin) [4]. Previous reports suggested that the components of the ECM, growth factors, cytokines and integrinrelated interactions can activate varied signaling cascades such as MAPKs, PI3K/Akt, Wnt/bcatenin, Ras, integrin-linked kinase and focal adhesion kinase that promotes EMT [5-8]. The activation of specific signaling pathways, thereby, converges toward a panel of transcriptional repressors, such as Snail, Zeb1, Slug [9-11]. Despite the recent progress in understanding the molecular maneuvers in EMT, the detailed analysis still remains uncharacterized.

SIRT1 belongs to the mammalian sirtuin family and are implicated in a range of agerelated diseases [12]. The physiological functions of SIRT1 are linked to its enzymatic activity as a lysine deacetylase. However, the precise role of SIRT1 in tumorigenesis is obscure and continually being contested $[13,14]$. Numerous reports suggest SIRT1 as a tumor suppressor that protects from oncogenic stress $[15,16]$. Mice with a heterozygotic deletion of the Sirt1 allele in a Tp53 haploinsufficient background showed increased genomic instability and develop tumors [17]. In addition, SIRT1 deacetylates and destabilizes the protooncogene Myc and prevent tumor progression [18]. On other hand, it was also reported that SIRT1 activation reduces apoptosis and promotes survival of the cancer cells, suggesting that SIRT1 could function, at least under certain circumstances, as a tumor-promoting factor $[19,20]$. However, the plausible function and regulation of SIRT1 towards the mesenchymal switch and tumor progression in context of OC still remains elusive. Thereby, in the present study, we identified the detailed mechanism underlying the role and regulation of SIRT1 expression towards the progression of ovarian cancer.

\section{Materials and Methods}

\section{Cell culture and treatments}

Human ovarian adenocarcinoma and teratocarcinoma cell lines SKOV-3 and PA-1 (ATCC, USA) were maintained in RPMI1640 and MEM respectively, all supplemented with 10\% fetal bovine serum (FBS), 100 $\mu \mathrm{g} / \mathrm{ml}$ streptomycin and $100 \mathrm{U} / \mathrm{ml}$ penicillin (Invitrogen). Human immortalized ovarian surface epithelial cells, IOSE (a kind gift from Drs. N. Aueresperg and Clara Salamanca, Vancouver, Canada) was maintained in 1:1 ratio of MCDB105 (Sigma-Aldrich; USA)-Medium199 (Invitrogen) and supplemented as above. LPA (Sigma), LPA-receptor inhibitor (Pertussis toxin, PTX) and Resveratrol was used at a conc. of $20 \mu \mathrm{M}, 100 \mathrm{nM}$ and $10 \mu \mathrm{M}$ respectively, unless otherwise specified, Prior to each treatment, cells were serum-starved for 16 $\mathrm{h}$, followed by $1 \mathrm{~h}$ pre-treatment with inhibitors and then induced with LPA.

Plasmids, siRNA and transient transfections

The human SIRT1 expression vector Flag-SIRT1 and Flag-SIRT1 H363Y (deacetylase domain mutation) were purchased from Addgene (Cambridge, MA, USA). Transient transfections with $1 \mu$ g plasmid DNA were performed with Lipofectamine 2000 (Invitrogen) at 1:3 ratio for 24-48 h. SIRT1 siRNA (Santa Cruz Biotechnology, USA) were used at $20 \mathrm{nM} / 2.5 \times 10^{5}$ cells with Lipofectamine 2000 (Invitrogen) for $48 \mathrm{~h}$ [21].

Immunofluorescence microscopy (IF)

Immunofluorescence staining with E-cadherin and N-cadherin (1:100) antibodies followed by Alexafluor 488-conjugated secondary antibody was performed as described previously [22]. For phalloidin staining $10^{5}$ cells/well were treated and the cellular morphology was observed as mentioned previously [23].

RNA isolation and Q-PCR

Total RNA was isolated using TRI-reagent (Sigma) following the standard protocol. First-strand cDNA synthesis followed by Q-PCR assay was performed and analyzed as described previously [24]. The primer sequences are mentioned in Table 1. 


\begin{tabular}{|c|c|}
\hline Cellular Physiology & $\begin{array}{l}\text { Cell Physiol Biochem 2017;41:795-805 } \\
\text { DI. } 101159 \text { (100045874 }\end{array}$ \\
\hline and Biochemistry & 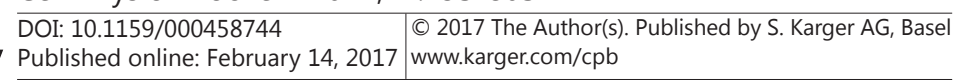 \\
\hline
\end{tabular}

Table 1. The primer sequences

\begin{tabular}{llll}
\hline Gene & Forward Primer & Reverse Primer & Tm \\
\hline $18 S r R N A$ & GATTCCGTGGGTGGTGGTGC & AAGAAGTTGGGGACGCCGA & 60 \\
CDH1 & GTCACTGACACCAACGATAATCCT & TTTCAGTGTGGTGATTACGACGTTA & 60 \\
CDH2 & CCATCAAGCCTGTGGGAATC & GCAGATCGGACCGGATACTG & 60 \\
CLDN7 & GTGGCAGATGAGCTCCTATGC & CATCCACAGCCCCTTGTACA & 60 \\
VIM & ACACCCTGCAATCTTTCAGACA & GATTCCACTTTGCGTTCAAGGT & 60 \\
SIRT1 & CGGGAATCCAAAGGATAATCA & CCTCGTACAGCTTCACAGTCAACT & 60 \\
ZEB1 & CAATGATCAGCCTCAATCTGCA & CCATTGGTGGTTGATCCCA & 60 \\
\hline
\end{tabular}

Western blot analysis

Cell lysis and protein extraction were performed as described previously [25] and subjected to immunoblotting with antibodies specific for the proteins including, ZEB1, HIF1 $\alpha$, GAPDH (Santa Cruz Biotechnology, USA); SIRT1, E-cadherin, Vimentin, Acetyl-lysine (Cell Signaling Technologies, USA). Secondary antibodies used were Horse Radish Peroxidase (HRP) tagged and was detected with ECL detection reagent (Bio-Rad, USA) by chemiluminesence.

\section{Cell invasion assay}

Cell invasion was studied using Matrigel invasion chamber (BD Biosciences, USA) following the protocol described earlier [26]. In brief, the cells were transiently transfected with SIRT1-WT/HY and SIRT1siRNA on previous day, allowed to recover overnight. The cells were then trypsinized, counted and equal number were added in the upper chamber and allowed to invade for $22 \mathrm{~h}$.

\section{Co-immunoprecipitation assay}

Whole cell protein was extracted and quantified [25]. Specific antibodies or pre-immune IgGs were incubated overnight with the cell lysates, before being absorbed by Protein A/G-plus Agarose beads (Sigma). Precipitated immune complex was then released by boiling with SDS-electrophoresis sample buffer (1X) followed by western blot analyses.

\section{MMP-9 activity by Gelatin zymography}

Post $24 \mathrm{~h}$ of transfection, cell media were collected and concentrated. The concentrated medium was then subjected to $0.1 \%$ gelatin- $10 \%$ SDS-PAGE as previously described [26]. Gels were stained with $0.1 \%$ Coomassie blue R250 and then destained followed by imaging.

\section{Immunofluorescence (IF)-based detection for Immunohistochemistry (IHC)}

The Department of Pathology, IPGMER-SSKM Hospital, Kolkata, India, provided us with blocks of ovarian cancer and non-cancer tissue sample as archival materials to use for IHC study. The ethical clearance to perform the above-mentioned experiments was obtained from the authorized committee of CSIR-IICB and SSKM Hospital (Memo No. Inst/IEC/615). Sectioning was performed, processed and stained as mentioned earlier [22].

\section{Statistical analysis}

All data are expressed as mean \pm SEM and is represented by error bars. The statistical significance was done by two-tailed Student's t-test. $\mathrm{p}<0.05$ (denoted with *) was considered significant unless otherwise stated. The experiments were repeated at least three times in duplicate.

\section{Results}

Activation of SIRT1 prevents mesenchymal switch and invasion in OC cells

To address the pathophysiological significance of SIRT1 in OC, we first evaluated the role of SIRT1 in the EMT and invasion in vitro.

Overexpression of wild-type (WT) but not the enzyme-deficient (HY) SIRT1 attenuated the invasion of PA1 OC cells ( $\sim 1.8$ fold, ${ }^{*} p<0.05$; Fig. 1A-i, ii), however knockdown of SIRT1 promoted cell invasion ( $\sim 4$ fold, ${ }^{*} p<0.05$; Fig. 1B-i,ii). The activity of MMP-9, regulating the KARGER 
invasion phenomenon also gets abrogated upon SIRT1-WT overexpression (Fig. 1C). In addition, SIRT1-WT was found to up-regulate the epithelial gene E-cadherin (CDH1) and downregulate the mesenchymal gene Vimentine (VIM) expression (Fig. 1D-E), whereas suppression of SIRT1 showed the opposite effect in PA1 cells (Fig. 1F-G). Similar observations with increase in the E-cadherin expression ( 1.5 fold) upon SIRT1-WT overexpression were obtained, whereas SIRT1-knockdown showed reverse effect at the protein level (Fig. 1H-J). Increased expression of E-cadherin was further validated by immunofluorescence study in the WT but not the mutant (HY) SIRT1 overexpressed cells, where N-cadherin showed reversed results (Fig. 1K). Similar observations were recapitulated in PA1 cells treated with SIRT1 agonist (Resveratrol) and antagonist (Sirtinol) (Fig. 1L). Further analysis of the alterations in cellular morphology by TRITC-phalloidin staining of the treated cells revealed that upon inhibition of SIRT1 the non-cancer ovarian cells (IOSE) showed a mesenchymal phenotype (Fig. 1M). Treatment with resveratrol further showed increase in the epithelial genes $(\sim 4$ fold increase in E-cadherin expression, ${ }^{*} \mathrm{p}<0.05$; Fig. 1N-0). Together these data suggest that activation of SIRT1 contributes to attenuate EMT and invasion in OC cells.

\section{SIRT1 deacetylates and inactivates HIF1 $\alpha$}

To acquire an overview of the physiological consequences of SIRT1 in OC, we analyzed the interaction between SIRT1 and HIF1 $\alpha$. Earlier reports suggested that in colon cancer, SIRT1 modulates cellular responses to hypoxia by HIF1 $\alpha$ deacetylation [27].

HIF-1 $\alpha$ was found to co-precipitate with SIRT1 in both the SIRT1-WT overexpressed PA1 and SKOV3 OC cell lines (Fig. 2A-B). Furthermore, to analyze the functional significance of this observed interaction, we examined whether SIRT1 deacetylates HIF1 $\alpha$ in OC cells. Lysyl-acetylated HIF1 $\alpha$ was analyzed by immunoblotting with anti-acetyl-lysine, upon immunoprecipitation by anti-HIF1 $\alpha$. It was observed that acetylated level of HIF $1 \alpha$ decreases upon overexpression of SIRT1-WT in both the OC cell lines (Fig. 2C). The resulting deacetylation of HIF1 $\alpha$ was supposed to inhibit its binding with the co-activators to promote the downstream target gene expression. To this extent, we analyzed the expression status of a critical EMT-inducing transcription factor ZEB1, which was also reported as a downstream target of HIF1 $\alpha$ [28]. SIRT1-WT overexpression leads to attenuation at the expression level of ZEB1 ( 2 fold, $*$ p < 0.05) and vice versa (Fig. 2D). Similar changes were observed the protein level expression of ZEB1, whereas no such significant changes were observed in case of HIF1 $\alpha$ expression (Fig. 2E). Thus it can be proposed that SIRT1 modifies the functional role of HIF $1 \alpha$, thereby affecting the downstream events. Together, these data suggest that activation of SIRT1 inhibits HIF1 $\alpha$ target genes and thus leads to suppression of EMT in OC cells.

\section{LPA promotes EMT and invasion of OC cells by suppressing SIRT1}

Recent studies showed that in context of OC progression, LPA plays a significant function due to its high enrichment in the tumor microenvironment [29-31]. Based on the previously mentioned contributions of LPA towards increased invasiveness, we first assessed its effect towards the mesenchymal switch in the non-cancer ovarian epithelial cell line, IOSE. Exposure to LPA in these cells resulted in actin rearrangement resembling the mesenchymal phenotype as well as reduced the E-cadherin levels (Fig. 3A). In addition, LPA leads to enhanced invasion of PA1 OC cells $\left(\sim 3.5\right.$ fold, $\left.{ }^{*} p<0.05\right)$, which gets reduced in presence of its receptor inhibitor, PTX (Fig. 3B).

Since LPA induces EMT whereas, SIRT1 overexpression was found to show the reverse effect, we want to investigate whether there is any existing regulation of SIRT1 expression by LPA. We found significant down-regulation in the expression of SIRT1 at both transcriptional and translational levels upon treatment with LPA at two different doses $(\sim 2.2$ fold at $10 \mu \mathrm{M},{ }^{*} \mathrm{p}<0.05$; Fig. 3C-D). Further, no significant change in expression of SIRT1 was obtained in the LPA-exposed cells upon pre-treatment with LPA-receptor inhibitor (Fig. 3EF). To gain insight we assessed the expression levels of both epithelial and mesenchymal genes upon exposure to LPA and SIRT agonist. LPA leads to an increase in the expression

\section{KARGER}




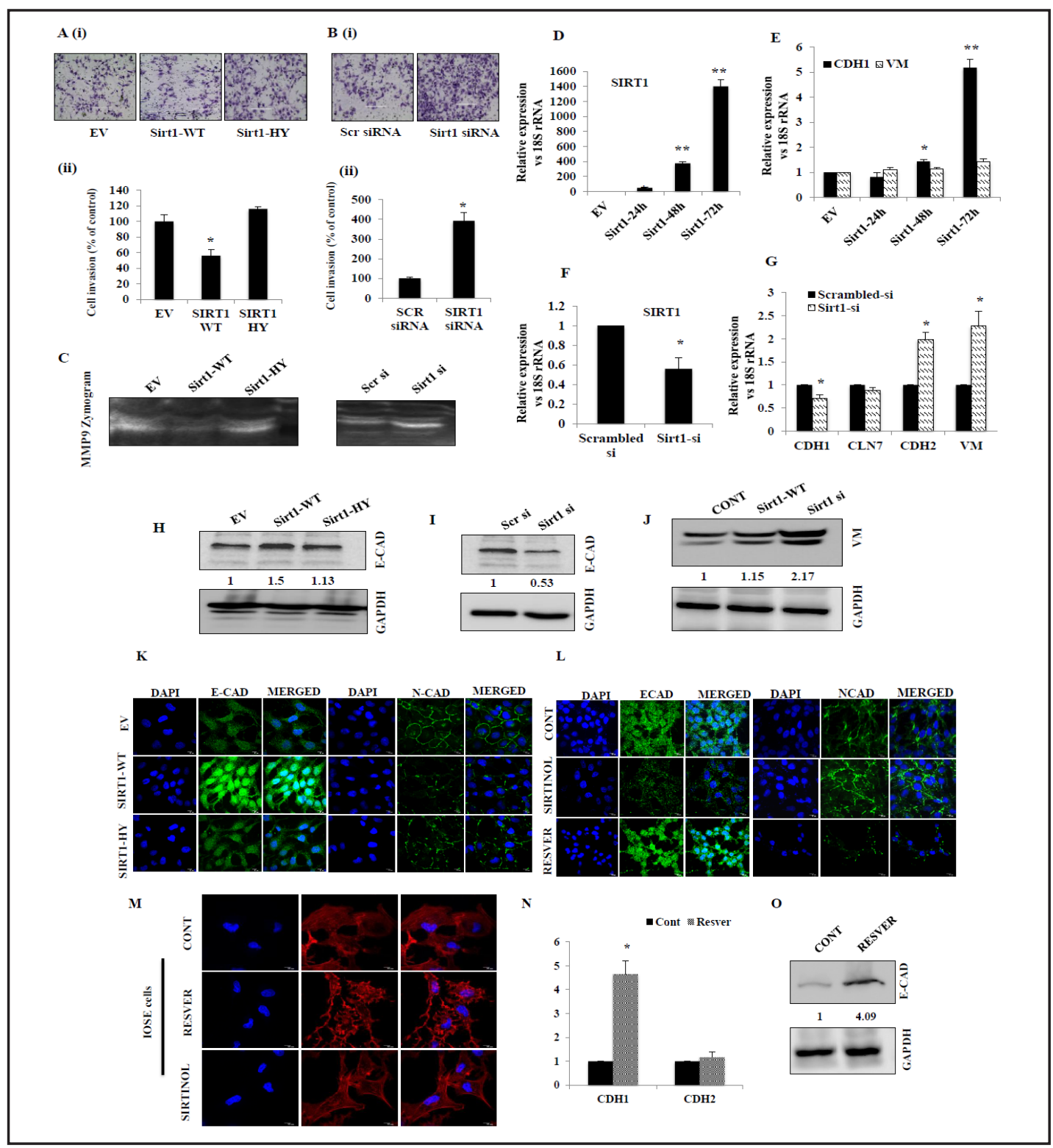

Fig. 1. SIRT1 activation represses OC invasion by inhibiting EMT. PA1 cells were transiently transfected with WT- or HY- SIRT1 expression constructs and SIRT1-siRNA. (A-B,i) Matrigel Invasion assays were performed and $(A-B, i i)$ cells at three independent fields for each well were counted and plotted $(* p<0.05)$. EV represents empty vector transfected control whereas Scr siRNA refers to non-targeted siRNA control. (C) Gelatin zymography for MMP-9 activity was performed in the transfected PA-1 cells as mentioned. (D) Overexpression of SIRT1 was confirmed at three different time points after transfection $\left(24,48\right.$ and $\left.72 \mathrm{hrs} ;{ }^{* *} \mathrm{p}<0.01\right)$. (E) Expression of epithelial (CDH1) and mesenchymal (VM) genes were measured by Q-PCR in these SIRT1 overexpressed cells $\left({ }^{*} \mathrm{p}<0.05,{ }^{* *} \mathrm{p}<0.01\right)$. (F) The efficiency of SIRT1-KD was measured by Q-PCR followed by (G) expression analysis of epithelial (CDH1, CLN7) and mesenchymal (CDH2, VM) genes (*p < 0.05). (H-I) Western analyses supporting similar results were performed. GAPDH is used as loading control. Densities of the respective bands are calculated by ImageJ software, normalized and fold change was mentioned. (K) Immunofluorescence study showing the effect of SIRT1 (WT/HY) on E-cadherin and N-cadherin levels was obtained. (L) Similar studies were performed when treated with SIRT1 agonist/antagonist, i.e. resveratrol and sirtinol respectively. (M) Morphological alterations were observed upon treatment with resveratrol and sirtinol by phalloidin staining of the actin in IOSE cells. (N-O) Expressions of the epithelial and mesenchymal genes were measured upon activation of SIRT1 by resveratrol $\left({ }^{*} \mathrm{p}<0.05\right)$.

\section{KARGER}




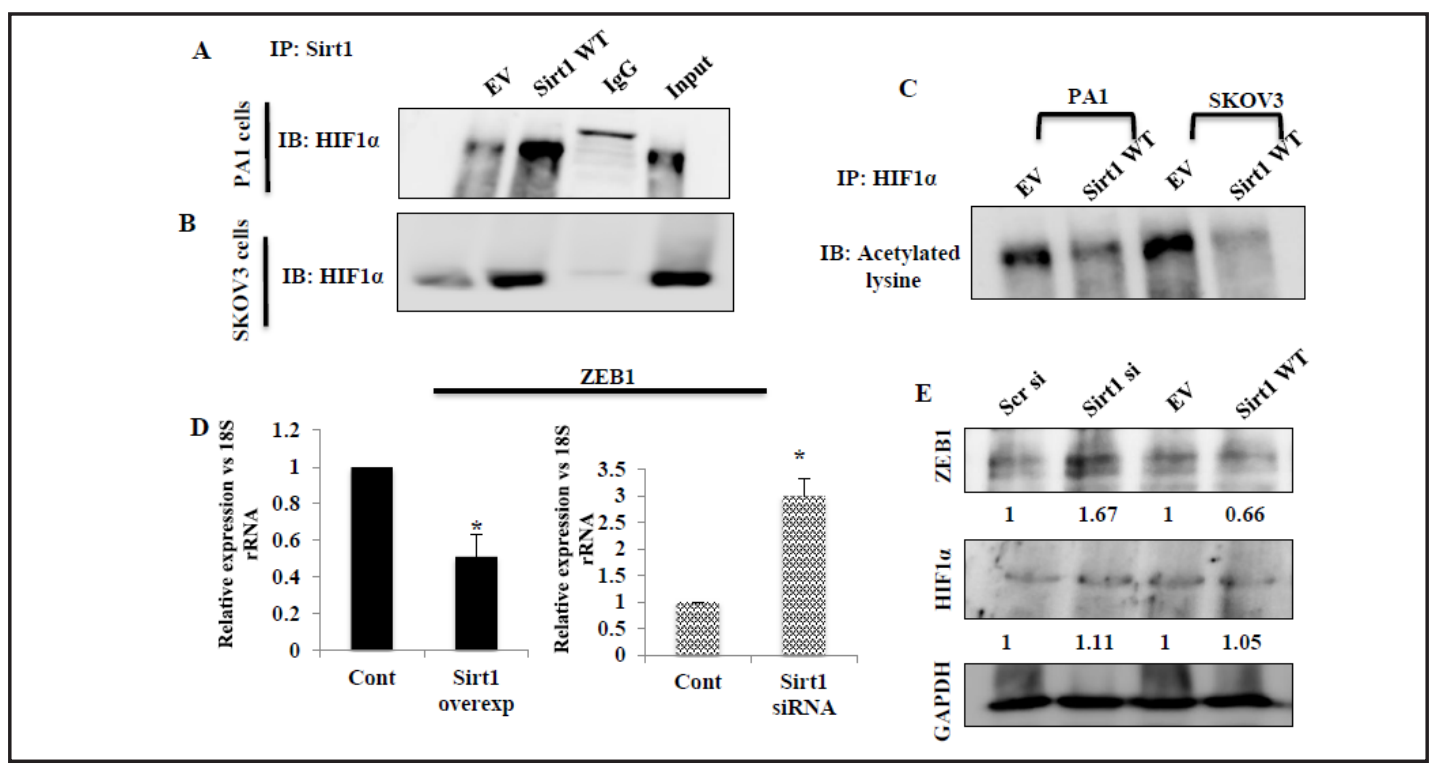

Fig. 2. SIRT1 deacetylates and blocks HIF1 $\alpha$ transcriptional activity. (A) PA1 and (B) SKOV3 cells were transfected with SIRT1-WT and empty vector (EV) plasmids and the cell extracts were immunoprecipitated with anti-SIRT1 and the co-precipitated HIF1 $\alpha$ was analyzed by western analysis. In both cases, IgG was used as a negative control. (C) Both the cell types were transfected with SIRT1-WT, the endogenous HIF1 $\alpha$ was immunoprecipitated and the acetylated HIF1 $\alpha$ was detected using anti-acetyl-lysine. (D) Expression of ZEB1 was evaluated upon overexpression and down-regulation of SIRT1 ( $\left.{ }^{*} p<0.05\right)$. (E) ZEB1 and HIF1 $\alpha$ expression at protein levels were further analyzed upon both overexpression and knockdown of SIRT1 by western analysis. Densities of the respective bands in western are calculated by ImageJ software, normalized and fold change was mentioned.

of mesenchymal gene CDH2 ( $\sim 2$ fold, $\left.{ }^{*} \mathrm{p}<0.05\right)$ whereas epithelial genes CDH1 and CLN7 get down regulated; however, activation of Sirt1 by Resveratrol-treatment showed reverse effect (Fig. 3G). Similar results were observed at the protein level expression of E-cadherin and Vimentine (Fig. 3H). Herein, we found that SIRT1 overexpression leads to inactivation of HIF1 $\alpha$ followed by reduced expression of ZEB1. Thereby, we further elucidate the functional significance of HIF $1 \alpha$ inactivation by SIRT1 in response to LPA. Increase in the expression of ZEB1 ( 1.23 fold) was observed when treated with LPA compared to non-treated cells. However, overexpression of SIRT1-WT reduces the LPA-induced expression of ZEB1 ( 2.5 fold), whereas, in case of enzyme deficient SIRT1-HY no such distinct changes were observed (Fig. 3I).

\section{SIRT1 expression is down regulated in ovarian cancer patients}

SIRT1 expression level was investigated in human OC tissue samples. Immunofluorescence imaging of the $\mathrm{OC}$ tissue sections (Fig. 4Aii, $\mathrm{n}=10$ ) showed attenuated expression of SIRT1 compared to normal ovarian tissue (Fig. 4Ai, $\mathrm{n}=10$ ). In addition, the tissue sections were stained with only secondary antibody and DAPI to show specificity of the staining (Fig. 4B). Taken together, this data confirms our in vitro observations, which relates the reduced expression of SIRT1 to the increased EMT/invasion phenomenon obtained in OC cells.

\section{Discussion}

Similar to numerous human pathologies like metabolic, cardiovascular and neurodegenerative disorders, cancer is also closely related to aging [32]. SIRT1, named as the 'longevity gene' was found to be an effective target in the treatment of several aging-related 


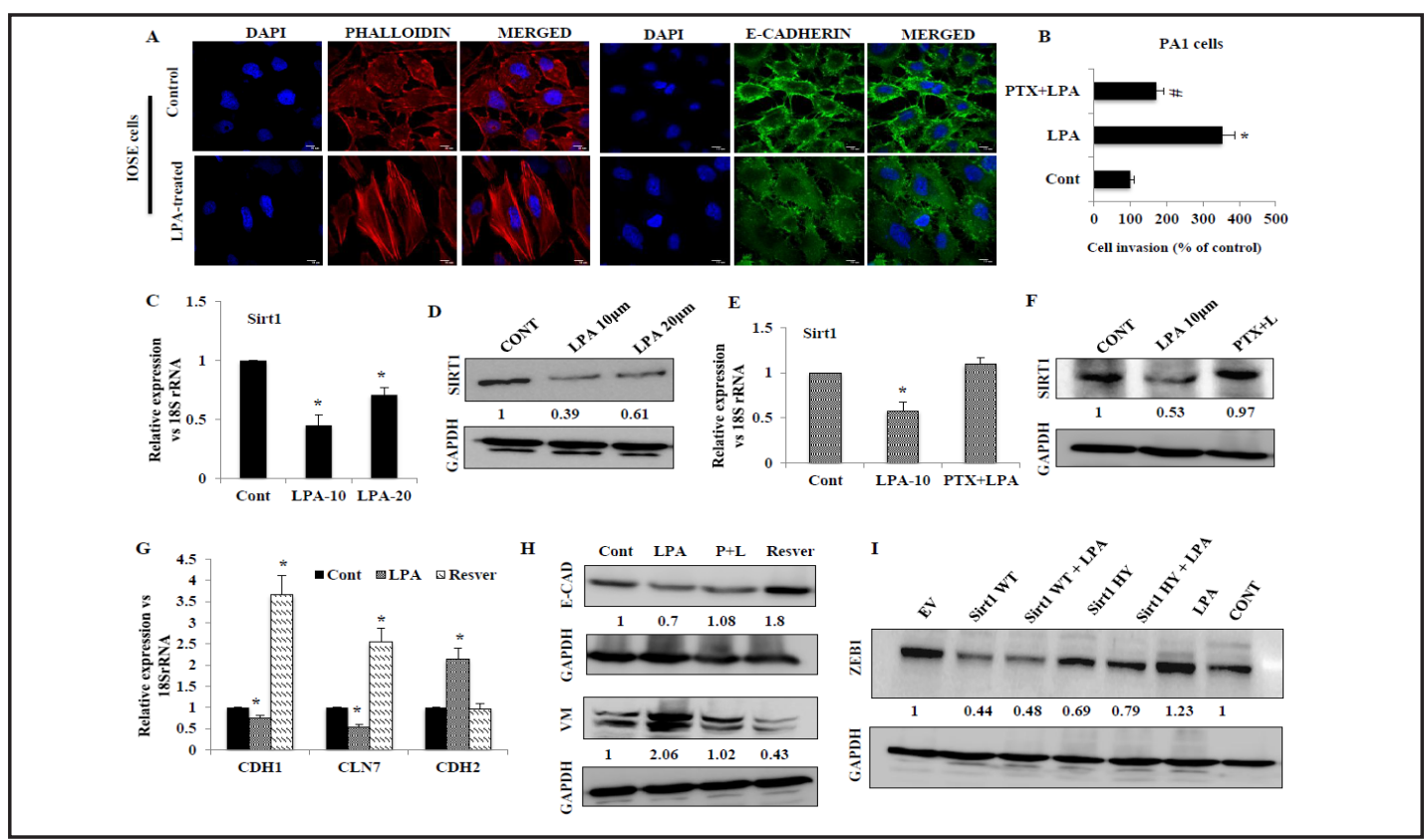

Fig. 3. LPA represses SIRT1 and promotes the mesenchymal switch in OC cells. (A) Rearrangement of actin filaments by TRITC-phalloidin staining and E-cadherin expression analysis upon exposure to LPA in IOSE cells. Scale bar,10 $\mu \mathrm{m}$. (B) Invasion analysis in PA1 OC cells upon LPA treatment in presence/absence of its receptor inhibitor PTX $\left({ }^{*} \mathrm{p}<0.05\right.$, cont vs LPA; \# $<<0.05$, LPA vs PTX+LPA). (C-D) SIRT1 expression analysis was performed with the indicated doses of LPA $(0,10$ and $20 \mu \mathrm{M})$, both at RNA $\left({ }^{*} p<0.05\right)$ and protein levels. (E-F) Expression of SIRT1 was analyzed by both Q-PCR $\left({ }^{*} \mathrm{p}<0.05\right)$ and immunoblot assay upon treatment of PA-1 cells with LPA in absence/presence of PTX. GAPDH was used as loading control. (G) Expression analysis of epithelial and mesenchymal genes were performed at both the RNA $\left({ }^{*} \mathrm{p}<0.05\right)$ and $(\mathrm{H})$ protein levels upon treatment of PA1 cells with LPA and resveratrol. (I) ZEB1 expression status was observed on overexpression of WT/HY-SIRT1 in presence and absence of LPA. GAPDH was obtained as a loading control. In all the cases, the densities of the respective bands in western are calculated by Image J software, normalized to GAPDH and fold change was mentioned.

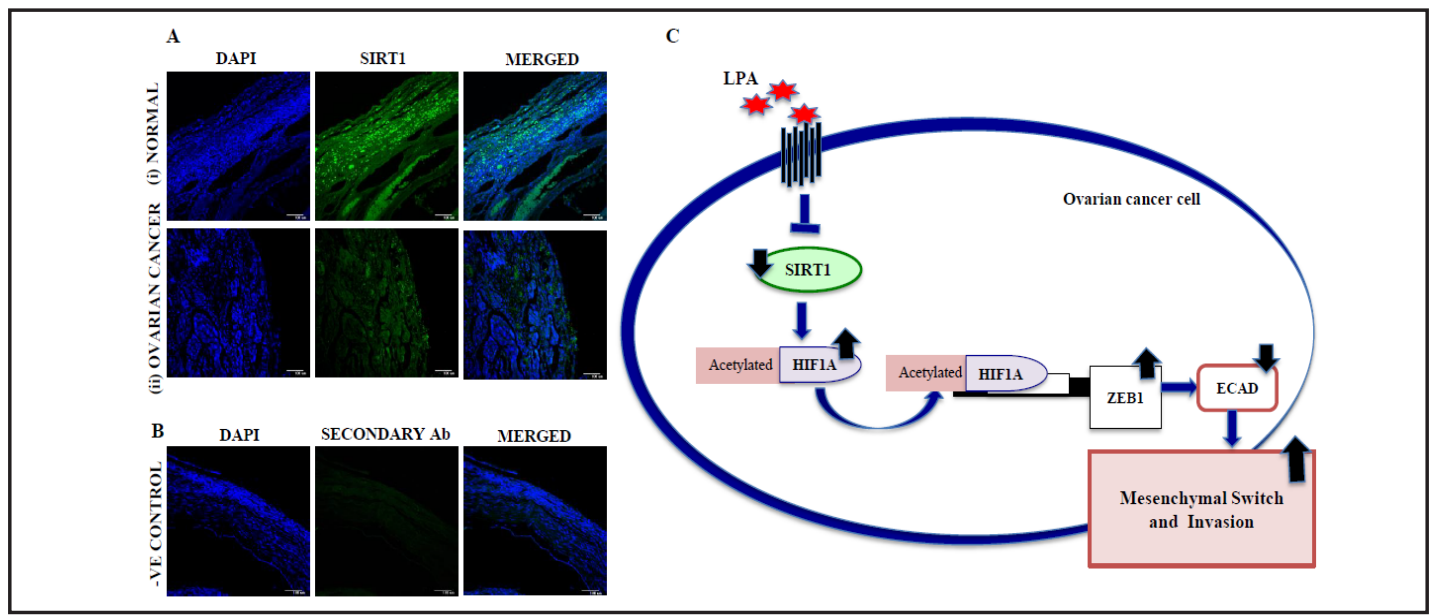

Fig. 4. Human ovarian tumor samples exhibit decreased SIRT1 expression. (A) Expression level of SIRT1 was shown by fluorescence-IHC in ovarian tissue sections of normal (i, $\mathrm{n}=10)$ and high-grade ovarian cancer patients ( $\mathrm{ii}, \mathrm{n}=10$ ). DAPI was used to stain the nucleus and merged images were provided. Scale bar, 100 $\mu \mathrm{m}$. (B) Tissue sections stained with only the secondary antibody was represented as negative control. Scale bar, $100 \mu \mathrm{m}$. (C) A model depicting that down-regulation of SIRT1 by LPA promotes epithelial-mesenchymal transition and eventually leads to cancer invasion.

\section{KARGER}


diseases like CHD, type-2 diabetes and Alzheimer [12,14]. However, the crucial contribution of SIRT1 towards carcinogenesis remains debatable, as reports suggests that SIRT1 can function both as a positive or negative regulator of tumorigenesis in different cancer types. High levels of SIRT1 were associated with poor prognosis mainly in prostate, lung and gastric carcinomas $[20,33,34]$. Additionally, SIRT1 was also reported to deactivate NF-kB and HIF1 $\alpha$ through deacetylation, thus suppressing cancer progression [27, 35]. However, one major downside of the previous studies is that, few have explored the regulators for SIRT1 expression in different cancer types.

Given that EMT represents the crucial event in the transition from pre-invasive to invasive phenotype, herein, we wanted to uncover the role and regulation of SIRT1 towards the mesenchymal switch in OC cells. We found that overexpression as well as activation of SIRT1 leads to attenuation in the mesenchymal switch but not the enzyme-deficient form (HY) in OC cells. Moreover, MMP-9 activity was found to decrease upon SIRT1 activation resulting in reduced invasion of OC cells. To gain an insight on the role of SIRT1 in suppressing EMT, we found that it interacts with, deacetylates and thereby inactivates HIF $1 \alpha$ transcriptional activity. Central EMT-inducing transcription factor ZEB1 was reported as a direct transcriptional repressor of the epithelial gene E-cadherin and one of the most potent inducer of mesenchymal switch in different physiological and pathological contexts [9-11]. To this extent, we obtained attenuated ZEB1 expression, which is a HIF1 $\alpha$ target gene, when SIRT1-WT was overexpressed. Our data thus suggests that SIRT1 activation in OC cells results in reduced expression of ZEB1 which, further promotes the maintenance of an epithelial phenotype, inhibiting cancer invasion.

Based on the importance of SIRT1 observed in suppressing EMT in OC cells, we further wanted to unveil the regulation of its expression in these cells. Till date, report showed that hypoxic stress attenuates SIRT1 expression at the transcriptional level in a SUMOylation-dependent manner to aid cancer metastasis [36]. However, no studies have explored any other regulation for SIRT1 expression in different cancer types. Previous reports in OC progression showed, LPA to play a critical role towards the cancer invasion/metastasis [29-30]. Since LPA was found to present at a significantly higher levels $(20-80 \mu \mathrm{M})$ in the ascitic fluid of OC patients, it is lately recommended as a biomarker for ovarian cancer [31]. Major biological function of LPA is mediated through activation of G-proteins coupled to a large family of cell surface receptors ( $\left.\mathrm{LPA}_{1}, \mathrm{LPA}_{2}, \mathrm{LPA}_{3}\right)$ [37]. Considering supporting evidences and our unpublished data, the enrichment of LPA in the microenvironment, might play a significant role in aggressiveness of OC cells [38-40]. Several evidences have implicated its role in promoting survival, invasion and metastasis through activation of matrix-metalloproteinases and varied oncogenic signaling cascades [38-43]. This prompted us to uncover the contribution of this metabolite towards the regulation of SIRT1, specifically in case of OC.

LPA was found to down-regulate the expression of SIRT1 in the OC cells, which was not observed when pre-treated with its receptor inhibitor. Moreover, LPA was found to promote the mesenchymal switch by up-regulating ZEB1 expression, which gets reversed upon SIRT1 overexpression. Thus, our data suggest that LPA induces EMT and invasion in OC cells by suppressing SIRT1 expression. According to the previous reports and our unpublished data, LPA induces the expression of HIF1 $\alpha$ promoting invasion in OC cells [44-46]. From our data, it can be suggested that LPA-mediated SIRT1 down-regulation helps in the maintenance of the acetylated and functional form of HIF $1 \alpha$ thereby, triggering cancer invasion. Together, LPA thus plays a central role in maintenance of both enhanced expression and activity of HIF $1 \alpha$, thereby contributing to the aggressive nature of OC. To validate the in vitro results, we found reduced expression of SIRT1 in the ovarian cancer tissue samples when compared to normal ovarian tissues.

In summary, our findings reinforce the notion that SIRT1 contributes to regulation of OC invasion by fine-tuning the EMT phenomenon. To this extent, LPA, which gets highly enriched in OC, promotes the epithelial to mesenchymal switching by repressing the expression of SIRT1. Thereby, development of small-molecule compounds that induces SIRT1 activity in OC will be helpful, both in understanding the mechanistic aspect of SIRT1 in cancer 
metastasis and benefiting patients with advanced-stage cancer. Additionally, targeting LPA activated pathways or its receptor, to increase the SIRT1 expression may yield novel therapeutic solutions against aggressiveness of ovarian cancer.

\section{Acknowledgments}

We thankfully acknowledge Council of Scientific and Industrial Research (CSIR, Project No. BSC-0101, BSC-0206), Govt. of India for funding this work. Technical assistance of Diptadeep Sarkar for carrying out all the confocal imaging is gratefully acknowledged. The technical assistance of Prabir Kumar Dey (CSIR-IICB) is gratefully acknowledged. Other members of SSR laboratory are thankfully acknowledged for their co-operation.

\section{Disclosure Statement}

The authors declare no conflicts of interest.

\section{References}

1 Siegel R, Ma J, Zou Z, Jemal A: Cancer statistics. CA Cancer J Clin 2014;64:9-29.

2 Thiery JP, Sleeman JP: Complex networks orchestrate epithelial-mesenchymal transitions. Nat Rev Mol Cell Biol 2006;7:131-142.

3 Thiery JP, Acloque H, Huang RY, Nieto MA: Epithelial- mesenchymal transitions in development and disease. Cell 2009;139:871-890.

4 Savagner P: Leaving the neighborhood: molecular mechanisms involved during epithelial-mesenchymal transition. Bioessays 2001;23:912-923.

-5 Hu Q, Tong S, Zhao X, Ding W, Gou Y, Xu K, Sun C, Xia G: Periostin Mediates TGF- $\beta$-Induced Epithelial Mesenchymal Transition in Prostate Cancer Cells. Cell Physiol Biochem 2015;36:799-809.

-6 Haase VH: Oxygen regulates epithelial-to-mesenchymal transition: insights into molecular mechanisms and relevance to disease. Kidney Int 2009;76:492-499.

-7 Vincent T, Neve EP, Johnson JR, Kukalev A, Rojo F, Albanell J, Pietras K, Virtanen I, Philipson L, Leopold PL, Crystal RG, de Herreros AG, Moustakas A, Pettersson RF, Fuxe J: A SNAIL1-SMAD3/4 transcriptional repressor complex promotes TGF-beta mediated epithelial-mesenchymal transition. Nat Cell Biol 2009;11:943-950.

8 Li Y, Li Y, Zhang J, Zheng C, Zhu H, Yu H, L Fan L: Circulating Insulin-Like Growth Factor-1 Level and Ovarian Cancer Risk. Cell Physiol Biochem 2016;38:589-597.

9 Vandewalle C, Van Roy F, Berx G: The role of the ZEB family of transcription factors in development and disease. Cell Mol Life Sci 2009;66:773-787.

10 Sanchez-Tillo E, Lazaro A, Torrent R, M, Vaquero EC, Castells A, Engel P, Postigo A: ZEB1 represses E-cadherin and induces an EMT by recruiting the SWI/SNF chromatin- remodeling protein BRG1. Oncogene 2010;29:3490-3500.

-11 Song N, Liu H, Ma X, Zhang S: Placental Growth Factor Promotes Ovarian Cancer Cell Invasion via ZEB2. Cell Physiol Biochem 2016;38:351-358.

-12 Guarente L: Sirtuins in aging and disease. Cold Spring Harb Symp Quant Biol 2007;72:483-488.

13 Liu T, Liu PY, Marshall GM: The critical role of the class III histone deacetylase SIRT1 in cancer. Cancer Res 2009;69:1702-1705.

14 Saunders LR, Verdin E: Sirtuins: critical regulators at the crossroads between cancer and aging. Oncogene 2007;26:5489-5504.

15 Simic P, Williams EO, Bell EL, Gong JJ, Bonkowski M, Guarente L: SIRT1 suppresses the epithelial-tomesenchymal transition in cancer metastasis and organ fibrosis. Cell Rep 2013;3:1175-1186.

16 Firestein R, Blander G, Michan S, Oberdoerffer P, Ogino S, Campbell J, Bhimavarapu A, Luikenhuis S, de Cabo R, Fuchs C, Hahn WC, Guarente LP, Sinclair DA: The SIRT1 deacetylase suppresses intestinal tumorigenesis and colon cancer growth. PLoS One 2008;3:e2020. 


\section{Cellular Physiology Cell Physiol Biochem 2017;41:795-805

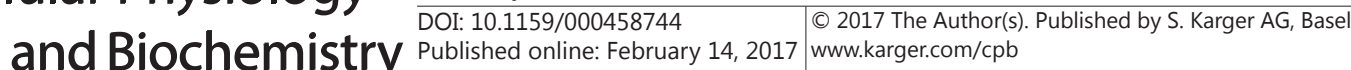

Ray et al.: LPA Suppresses SIRT1 Expression

17 Wang RH, Sengupta K, Li C, Kim HS, Cao L, Xiao C, Kim S, Xu X, Zheng Y, Chilton B, Jia R, Zheng ZM, Appella E, Wang XW, Ried T, Deng CX: Impaired DNA damage response, genome instability, and tumorigenesis in SIRT1 mutant mice. Cancer Cell 2008;14:312-323.

18 Yuan J, Minter-Dykhouse K, Lou Z: A c-Myc-SIRT1 feedback loop regulates cell growth and transformation. J Cell Biol 2009;185:203-211.

19 Chen WY, Wang DH, Yen RC, Luo J, Gu W, Baylin SB: Tumor suppressor HIC1 directly regulates SIRT1 to modulate p53-dependent DNA- damage responses. Cell 2005;123:437-448.

20 Nakane K, Fujita Y, Terazawa R, Atsumi Y, Kato T, Nozawa Y, Deguchi T, Ito M: Inhibition of cortactin and SIRT1 expression attenuates migration and invasion of prostate cancer DU145 cells. Int J Urol 2012;19:1442-2042.

-21 Basu M, Mukhopadhyay S, Chatterjee U, Roy SS: FGF16 promotes invasive behavior of SKOV-3 ovarian cancer cells through the activation of MAPK signaling pathway. J Biol Chem 2014;289:1415-1428.

22 Basu M. Bhattacharya R, Ray U, Roy SS: Invasion of ovarian cancer cells is induced by PITX2-mediated activation of TGF- $\beta$ and Activin-A. Mol Cancer 2015;14:162.

23 Faulstich H, Trischmann, H, Mayer D. Preparation of tetramethyl-rhodaminyl phalloidin and uptake of the toxin into short-term cultured hepatocytes by endocytosis. Exp Cell Res 1983;144:73-82.

24 Bhattacharjee S, Das N, Mandala A, Mukhopadhyay S, Roy SS: Fenofibrate Reverses Palmitate Induced Impairment in Glucose Uptake in Skeletal Muscle Cells by Preventing Cytosolic Ceramide Accumulation. Cell Physiol Biochem 2015;37:1315-1328.

25 Nandi SS, Ghosh P, Sankar Roy SS: Expression of PITX2 Homeodomain Transcription Factor during Rat Gonadal Development in a Sexually Dimorphic Manner Cell Physiol Biochem 2011;27:159-170.

-26 Ghosh S, Basu M, Roy SS: ETS-1 protein regulates vascular endothelial growth factor-induced matrix metalloproteinase-9 and matrix metalloproteinase-13 expression in human ovarian carcinoma cell SKOV3. J Biol Chem 2012;287:15001-15015.

27 Lim JH, Lee YM, Chun YS, Chen J, Kim JE, Park JW: Sirtuin 1 modulates cellular responses to hypoxia by deacetylating hypoxia-inducible factor 1alpha. Mol Cell 2010;38:864-878.

28 Zhang W, Shi X, Peng Y, Wu M, Zhang P, Xie R, Wu Y, Yan Q, Liu S, Wang J: HIF-1 $\alpha$ Promotes EpithelialMesenchymal Transition and Metastasis through Direct Regulation of ZEB1 in Colorectal Cancer. PLoS ONE 2015;10:e0129603.

-29 Sengupta S, Wang Z, Tipps R, Xu Y: Biology of LPA in health and disease. Semin Cell Dev Biol 2004;15:503512.

30 Mills GB, Moolenaar WH: The emerging role of lysophosphatidic acid in cancer. Nat Rev Cancer 2003;3:582-591.

-31 Xu Y, Shen Z, Wiper DW, Wu M, Morton E, Elson P, Kennedy AW, Belinson J, Markman M, Casey G: Lysophosphatidic acid as a potential biomarker for ovarian and other gynecologic cancers. JAMA 1998;280:719-723.

- 32 Herranz D, Munoz-Martin M, Canamero M, Mulero F, Martinez-Pastor B, Fernandez-Capetillo O, Serrano M: Sirt1 improves healthy ageing and protects from metabolic syndrome-associated cancer. Nat Commun 2010;1:3.

33 Byles V, Zhu L, Lovaas JD, Chmilewski LK, Wang J, Faller DV, Dai Y: SIRT1 induces EMT by cooperating with EMT transcription factors and enhances prostate cancer cell migration and metastasis. Oncogene 2012;31:4619-4629.

-34 Cha EJ, Noh SJ, Kwon KS, Kim CY, Park BH, Park HS, Lee H, Chung MJ, Kang MJ, Lee DG, Moon WS, Jang KY: Expression of DBC1 and SIRT1 is associated with poor prognosis of gastric carcinoma. Clin Cancer Res 2009;15:4453-4459.

-35 Yeung F, Hoberg JE, Ramsey CS, Keller MD, Jones DR, Frye RA, Mayo MW: Modulation of NF-kappaBdependent transcription and cell survival by the SIRT1 deacetylase. EMBO J 2004;23:2369-2380.

-36 Sun L, Li H, Chen J, Dehennaut V, Zhao Y, Yang Y, Iwasaki Y, Kahn-Perles B, Leprince D, Chen Q, Shen A, Xu Y: A SUMOylation-dependent pathway regulates SIRT1 transcription and lung cancer metastasis. J Natl Cancer Inst 2013;105:887-898.

37 Noguchi K, Herr D, Mutoh T, Chun J: Lysophosphatidic acid (LPA) and its receptors. Curr Opin Pharmacol 2009;9:15-23.

-38 Fishman DA, Liu Y, Ellerbroek SM, Stack MS: Lysophosphatidic acid promotes matrix metalloproteinase (MMP) activation and MMP-dependent invasion in ovarian cancer cells. Cancer Res 2001;61:3194-3199. 


\section{Cellular Physiology Cell Physiol Biochem 2017;41:795-805 and Biochemistry DOI: 10.1159/000458744 14, 2017 O 2017 The Author(s). Published by S. Karger AG, Basel

Ray et al.: LPA Suppresses SIRT1 Expression

-39 So J, Navari J, Wang FQ, Fishman DA: Lysophosphatidic acid enhances epithelial ovarian carcinoma invasion through the increased expression of interleukin-8. Gynecol Oncol 2004;95;314-322.

- 40 Jeong KJ, Park SY, Cho KH, Sohn JS, Lee J, Kim YK, Kang J, Park CG, Han JW, Lee HY: The Rho/ROCK pathway for lysophosphatidic acid-induced proteolytic enzyme expression and ovarian cancer cell invasion. Oncogene 2012;31:4279-4289.

-41 Leve F, Peres-Moreira RJ, Binato R, Abdelhay E, Morgado-Díaz JA: LPA induces colon cancer cell proliferation through a cooperation between the ROCK and STAT-3 Pathways. Plos one 2015;10:e0139094.

-42 Park SY, Jeong KJ, Panupinthu N, Yu S, Lee J, Han JW, Kim JM, Lee JS, Kang J, Park CG, Mills GB, Lee HY: Lysophosphatidic acid augments human hepatocellular carcinoma cell invasion through LPA1 receptor and MMP-9 expression. Oncogene 2011;30:1351-1359.

43 Fan Q, Cai Q, Xu Y: FOXM1 is a downstream target of LPA and YAP oncogenic signaling pathways in highgrade serous ovarian cancer. Oncotarget 2015;6:27688-27699.

-44 Lee J, Park SY, Lee EK, Park CG, Chung HC, Rha SY, Kim YK, Bae GU, Kim BK, Han JW, Lee HY: Activation of hypoxia-inducible factor-1alpha is necessary for lysophosphatidic acid-induced vascular endothelial growth factor expression. Clin Cancer Res 2006;12:6351-6358.

45 Yang, K, Zheng D, Deng X, Bai L, Xu Y, Cong YS: Lysophosphatidic acid activates telomerase in ovarian cancer cells through hypoxia-inducible factor-1a and the PI3K pathway. J Cell Biochem 2008;105:11941201.

- 46 Ha JH, Ward JD, Radhakrishnan R, Jayaraman M, Song YS, Dhanasekaran DN: Lysophosphatidic acid stimulates epithelial to mesenchymal transition marker Slug/Snail2 in ovarian cancer cells via Gai2, Src, and HIF1 $\alpha$ signaling nexus. Oncotarget 2016:9224. 